\title{
Séparation gravitaire de l'eau et des suspensions solides
}

PAR

\author{
P. Treille \\ Ancien Elève de l'Ecole Polytechnique \\ Docteur es-Sciences, Chargé de la Recherche \\ Société Degrémont
}

L'utilisation à grande échelle du recyclage de l'eau dans l'industrie, base de cette série de conférences, doit permettre effectivement d'économiser nos ressources en eau, à une époque où les besoins deviennent de plus en plus importants, avec une pollution de plus en plus grande et partout répandue.

Le recyclage de l'eau existe déjà, puisque notre principale source d'approvisionnement est constituée par les eaux de rivière, qui reçoivent, d'amont en aval, les effluents de nombreuses communautés urbaines ou industrielles:

En effet, l'eau n'est pas consommée: elle est utilisée avant tout comme moyen de transfert, de transport, de lavage (chaleur, minerais, résidus de rinçage, etc.) et est rejetée après usage.

L'utilisation des eaux de surface ou celle des effuents d'une usine ne vont donc pas poser de problèmes fondamentalement différents, mais représenteront des variantes autour du même thème: ce dont nous disposons est un liquide aqueux; ce dont nous avons besoin est de l'eau propre, et, de toute façon, entre prélèvement et utilisation, il faut un traitement.

Le traitement le plus général - et qui constitue d'ailleurs la première phase sinon la phase unique de l'épuration est l'enlèvement des matières en suspension.

C'est sans aucun doute l'entraînement, par l'eau, de diverses substances solides qui constitue la partie la plus importante et la plus commune de la pollution. Cette partie solide doit être éliminée pour éviter d'autres inconvénients dont les principaux sont: bouchage de canalisations ou de réfrigérants, abrasion de pompes ou d'organes de mesure, usure de matériel divers, etc., avec de fortes incidences sur les coûts d'exploitation ou d'entretien des unités de fabrication.

Qu'il s'agisse donc de recyclage instantané ou de recyclage différé, les eaux utilisables sont polluées par la nature et par l'homme avec des substances organiques ou minérales en suspension, et, dans de nombreux cas, par des organismes vivants: plancton, bactéries ou virus.

La taille moyenne des particules varie très fortement, même pour un type donné de pollution.

Dans le tableau 1 sont répertoriés certains polluants avec leur dimension moyenne et l'ordre de grandeur du temps nécessaire pour que, sous l'influence de leur poids, ces particules parcourent verticalement un mètre d'eau.

Tableau 1

\begin{tabular}{|c|c|c|c|}
\hline & $\begin{array}{l}\text { Diamètre } \\
\text { LA PARTICULE: } \\
\quad(\mathrm{mm})\end{array}$ & ORDRE DE GRANDEUR & $\begin{array}{c}\text { TEMPS } \\
\text { DE DÉCANTATION } \\
\text { POUR } 1 \mathrm{~m}\end{array}$ \\
\hline & 10 & Gravier & 1 seconde \\
\hline & 1 & Sable & 10 secondes \\
\hline & 0,1 & Sable fin & 126 secondes \\
\hline & 0,01 & Argile & 110 minutes \\
\hline & 0,001 & Bactérie & 183 heures \\
\hline & 0,0001 & Part. colloïdale & 770 jours \\
\hline & 0,00001 & Part. colloïdale & 21 ans \\
\hline
\end{tabular}

En fait, un litre d'eau de bonne qualité peut contenir plusieurs dizaines de millions de particules de l'ordre du micron. Malgré leur nombre ces particules pèseront au total moins de $0,1 \mathrm{mg}$.

Il résulte de ces chiffres que la qualité de l'eau est une notion relative et qu'il est nécessaire, en fonction des besoins, de préciser quelle pollution résiduelle il est possible d'admettre et, bien entendu, il faut fixer des méthodes de mesure, en liaison avec les deux critères de qualité norma- 
lement employés pour l'objet de cette réunion: la concentration des matières en suspension et la turbidité de l'eau qui traduit la présence de particules de petite taille non visibles individuellement.

\section{TENEUR EN MATIËRES EN SUSPENSION}

Les méthodes employées en Europe sont très variables et peuvent donner des résultats fort différents, surtout sur les effluents filtrés, mais la France a été un des premiers pays à créer récemment une normalisation de la teneur en matières en suspension par la norme AFNOR T 90-105 de mars 1972.

Celle-ci prévoit trois méthodes :

- une filtration sur disques filtrants en fibres de verre qui peuvent par exemple être du type :

- Millipore ....... A P 20

- Sartorius ...... F N 501

- Durieux ...... D 28

- Schleicher ...... SS 6

- etc.

- une filtration sur couche d'amiante, de conduite et de reproductibilité assez délicates et risquant d'être mal réalisée pour les opérations de contrôle d'eaux;

- une centrifugation (accélération moyenne de $3000 \mathrm{~g}$ ), utilisable au cas où les méthodes par filtration ne sont pas applicables par suite du colmatage des filtres précédents, ce qui peut se produire notamment si la durée de filtration doit être supérieure à 1 heure.

Le recours à ces méthodes est nécessaire pour le contrôle d'eaux potables, d'eaux d'appoint divers et de rejets d'eaux résiduaires.

Il est limité par la précision des balances et la difficulté de filtrer de trop grands débits. Cependant, ces méthodes et tout particulièrement les disques filtrants dont la finesse théorique est de l'ordre de 1,5 micron, retiennent la totalité des matières en suspension avec les collö̈des, ce qui correspond à l'efficacité des traitements utilisés pour les types d'eaux précédemment cités.

Par contre, on réalise souvent des filtrations simples qui n'ont pas pour but d'éliminer les matières colloïdales et pour lesquelles on désire une méthode de contrôle rapide et ne titrant que les matières en suspension non colloïdales : ceci est le cas de beaucoup d'eaux de réfrigération ou d'arrosage.

On recherchera alors l'emploi de disques filtrants de porosité supérieure correspondant à la zone d'efficacité moindre souhaitée pour le traitement. Un tel emploi courant a évidemment un but comparatif et fonctionnel caractérisant l'efficacité relative d'une installation d'épuration.

\section{TURBIDITÉ}

Elle peut être, en pratique, déterminée de deux façons:

- En unités Jackson, avec étalonnage par une suspension témoin obtenue par mélange de sulfate d'hydrazine et d'hexaméthylène tétramine produisant de la Formazine.

Cette méthode est très employée internationalement. A titre d'exemple, l'eau de Seine présente couramment une turbidité d'une vingtaine d'U.J., alors qu'à travers le monde les normes pour eau potable peuvent imposer des turbidités variables de 0,1 à 5 U.J.
- En gouttes de mastic, suivant la méthode Dienert et Guillard, par ajout de gouttes d'une solution alcoolique cle mastic végétal dans une eau témoin parfaitement limpide. Cette méthode est surtout employée en France, mais n'est utilisable que jusqu'à 10 à 15 gouttes de mastic et concerne donc l'étude des eaux traitées et non celle des eaux brutes. La correspondance très irrégulière avec les unités Jackson est de lordre de 10 à 15 gouttes de mastic par unité.

Au point de vue corrélation entre les unités Jackson et la teneur en M.E.S., il est important de noter que, s'il y a un certain parallélisme de valeurs mesurées sur les eaux brutes, celui-ci n'existe plus que rarement sur les eaux épurées.

\section{Principaux modes d'épuration}

L'extraction des particules solides hors d'un liquide peut se faire selon deux principes différents :

- par simple action de la pesanteur, vers le haut (flottation) ou vers le bas (décantation), processus faisant appel à la grosseur et au poids spécifique des solides; - par filtration ou tamisage.

\section{DÉCANTATION}

Lorsqu'une particule est laissée à elle-même dans un liquide au repos, elle est soumise à une force motrice, la pesanteur, et à une force résistante, résultante des forces de viscosité et d'inertie. Quand on lâche cette particule avec une vitesse nulle, elle accélère, puis prend très rapidement une vitesse pratiquement constante. Diverses formules rendent compte du phénomène, en particulier celle de Stockes.

La vitesse réelle de décantation est la différence entre cette vitesse limite et la composante verticale de la vitesse du courant d'eau. D'où la limitation du débit de l'eau à traiter.

En fait, compte tenu de la variété des polluants à éliminer en même temps, la détermination des caractéristiques d'un décanteur résulte de l'étude expérimentale de l'eau, appuyée par une solide expérience.

\section{FLOTTATION}

Une bonne partie de la pollution industrielle est constituée par des corps de la chimie organique, donc légers. Il est plus facile de les alléger, à l'aide de bulles d'air spécialement injectées dans l'eau pour les faire flotter en surface, que de les alourdir artificiellement pour obtenir une vitesse de décantation raisonnable.

La flottation est donc devenue un mode de traitement très utilisé dans l'épuration moderne et dans le recyclage non seulement de l'eau, mais aussi des produits valorisables.

De nombreux exemples seront donnés dans les conférences suivantes: précisons simplement que, si la décantation d'un effluent est difficilement réalisable à plus de $2 \mathrm{~m}^{3} / \mathrm{m}^{2}$. h, la flottation sera aisée à plus de $5 \mathrm{~m}^{3} / \mathrm{m}^{2}$. h. On voit tout l'intérêt du système. Par ailleurs, la flottation fournit en général un résidu nettement plus déshydraté, donc moins cher à éliminer dans le traitement des résidus qui accompagne, très souvent, dans les conditions modernes d'environnement, l'épuration de l'eau. 


\section{FILTRATION}

Elle s'effectue en faisant passer l'eau à travers une matière poreuse. Cette matière peut agir de trois façons:

- en retenant mécaniquement les matières solides, contenues dans l'eau, dont le volume est trop important pour qu'elles traversent les pores de la matière poreuse;

- en servant de support à des microorganismes (principalement des algues) qui secrètent des diastases exerçant une action coagulante sur l'eau;

- en fixant par adsorption les matières contenues dans l'eau.

Il en résulte qu'il est difficile de définir une finesse de filtration qui est aussi variable suivant l'état d'encrassement du filtre.

Le contrôle pratique de l'efficacité d'une filtration peut certes être fait par comptage ou pesée des particules retenues sur des membranes témoin traitant le filtrat, mais, là encore, un processus de coagulation et d'autofiltration peut se réaliser et se traduire par l'interprétation erronée d'une granulométrie plus élevée que celle réelle à l'entrée du filtre témoin.

Néanmoins - et bien sûr arbitrairement — on distingue trois échelles de finesse de filtration qui correspondent à des techniques différentes de mise en œuvre de la filtration :

- la macrofiltration qui concerne des particules de 5 microns/20 microns, à 100,200 microns, quelques millimètres exceptionnellement;

- la microfiltration qui concerne des particules de $10 \mathrm{mi}$ crons à $0,3 / 0,5$ micron, c'est-à-dire à la limite du discernable au microscope ordinaire;

- l'ultrafiltration qui concerne des particules de moins de 0,3 micron et se trouve industriellement concrétisée par l'emploi des membranes, dites d'ultrafiltration et d'osmose inverse, membranes semi-perméables à travers lesquelles les processus de séparation font intervenir d'autres critères que la taille et sont, en pratique, caractérisés par leur pouvoir de coupure, c'est-à-dire par la masse moléculaire minimale des macromolécules totalement arrêtées.

C'est en fixant par adsorption les matières solides en suspension dans l'eau que les filtres exercent leur action essentielle.

La rétention des matières solides contenues dans l'eau provoque une obstruction progressive des interstices existant entre les éléments constitutifs de la matière poreuse. $\mathrm{Ce}$ phénomène est désigné sous le nom de colmatage du filtre. A mesure que ce colmatage se produit, les pertes de charge subies par le courant d'eau traversant le filtre s'accroissent.

La rapidité du colmatage dépend:

- de la nature des eaux traitées; elle est d'autant plus rapide que ces eaux sont plus chargées;

- du débit par unité de surface filtrante, ou vitesse de filtration; elle s'accroît avec cette vitesse;

- de la granulométrie de la matière filtrante; le colmatage d'une matière filtrante est d'autant plus rapide que ses éléments constitutifs sont plus fins.

Au cours de la filtration, on constate que le colmatage de la matière poreuse se produit à partir de la surface d'entrée de l'eau brute et pénètre progressivement en profondeur.
Ces considérations conduisent, d'une façon générale, à procéder à la filtration à travers une masse filtrante épaisse, constituée par des sables ou graviers sur une épaisseur de $0,60 \mathrm{~m}$ à $1,50 \mathrm{~m}$.

Un des principaux problèmes qui se posent consiste à assurer un lavage à la fois commode, efficace et économique de cette masse filtrante. Ce lavage exige que les éléments constitutifs de la masse filtrante soient préalablement détassés. Les crasses qui enrobent les éléments doivent ensuite en être mécaniquement détachées. Enfin, ces crasses doivent être entraînées par rinçage de la masse filtrante.

Ce rinçage s'effectue toujours par un courant d'eau.

Quant au détassage et au décrassage des éléments de la masse filtrante, ils s'effectuent, le plus souvent, soit en faisant circuler simultanément de l'air surpressé et de l'eau. L'eau seule suffit lorsque İe sable est très fin, mais l'air est indispensable dès que sa granulométrie est plus importante, car, sans l'aide de l'air comprimé, le débit d'eau nécessaire pour mettre le sable en suspension dans l'eau deviendrait vite prohibitif.

Le bon fonctionnement d'un filtre à sable dépend, évidemment, aussi bien en ce qui concerne son action de filtration proprement dite que son lavage, de la parfaite répartition, à travers la masse filtrante, des filets d'eau à filtrer et d'eau (et éventuellement d'air) de lavage. Le mode de collecte de l'eau (pendant la filtration) et sa répartition (pendant le lavage) à la base de la masse filtrante a donc une importance déterminante sur l'efficacité d'un filtre.

\section{Traitement particulier des colloïdes}

Les trois principes précédemment énoncés ne peuvent être mis en œuvre directement pour l'élimination des particules colloïdales :

- d'une part, le tableau 1 met en évidence leur très faible vitesse de décantation naturelle qui imposerait des temps de séjour incompatibles avec les possibilités pratiques d'installation de stations d'épuration;

- d'autre part, la filtration devient de plus en plus coûteuse à réaliser, même sans tenir compte de la présence, généralement simultanée, de particules beaucoup plus grossières, qui complique le problème de l'épuration.

Il est donc nécessaire de changer au préalable l'état des particules colloïdales : pour l'une ou l'autre des possibilités citées ci-dessus, le changement le plus intéressant est l'agglomération des particules entre elles pour augmenter très fortement la taille finale des corpuscules à éliminer.

Ce grossissement ne se fait pas naturellement; c'est dire qu'il existe des forces dont l'action est spécifique d'une suspension colloïdale et qui la maintiennent à l'état dispersé au cours du temps avec une remarquable stabilité.

\section{FACTEURS DE STABILITÉ}

En ce qui concerne les substances hydrophiles, la stabilité est assurée par le phénomène de l'hydratation, dans laquelle des molécules d'eau sont attirées à la surface des particules et forment une barrière à la mise en contact de deux particules.

Pour les substances hydrophobes, la théorie généralement admise est celle dite de «la double couche »: la surface 
de la particule est une couche chargée électriquement, et il existe une enveloppe d'ions chargés du signe opposé entourant la première couche. En simplifiant les nombreuses théories qui existent à ce sujet, et dont la première est due à Helmholtz (1879), on peut dire que la plupart des substances hydrophobes présentes en suspension colloïdale stable sont chargées, à leur surface, de charges électriques négatives.

Ces charges peuvent provenir d'ionisation de certains atomes à la surface de la particule par le remplacement de certains éléments de la structure cristalline de la particule par des éléments de signe différent. Elles peuvent également provenir d'une adsorption d'ions provenant de l'eau ellemême, et tout particulièrement d'ions $\mathrm{OH}$. Ces ions sont fermement fixés à la surface de la particule, et attirent des ions de charge opposée, provenant du mélange d'ions positifs et négatifs contenus dans l'eau elle-même. Cette couche d'ions de charge opposée (appelés contre-ions) est maintenue près de la particule par des forces électrostatiques. L'agitation thermique des molécules d'eau conduit les «contre-ions» à former une couche diffuse qui s'étend depuis la particule jusque dans la solution.

Si l'on mesure le polentiel en s'éloignant de la particule, on constate une décroissance exponentielle de ce potentiel, qui s'annule éventuellement au moment où la concentration. en anion est égale à celle des cations.

On peut distinguer plusieurs zones dans la couche diffuse, En particulier, il existe une zone où les ions sont fortement attachés à la particule, et se déplacent en même temps qu'elle dans le liquide interstitiel : cette couche peut donc en réalité être considérée comme faisant partie de la particule.

Une conséquence de l'existence de ces couches est de créer, dans un liquide apparemment neutre, des régions chargées électriquement. Le travail électrostatique nécessaire pour amener une charge depuis le liquide interstitiel jusqu'à un point d'une de ces zones, mesure le potentiel de ce point. Le potentiel s'accroît rapidement lorsque l'on s'approche de la particule.

Le potentiel de la surface du colloïde est appelé le potentiel de Nernst. Pour certains cas particuliers de systèmes bien définis, on peut calculer ce potentiel, mais c'est impossible dans les eaux naturelles.

Le second potentiel intéressant est le potentiel zêta, et correspond à la séparation des ions du solvant, qui se déplacent en même temps que la particule, et des ions du solvant, qui se déplacent indépendamment de la particule. Cette séparation détermine en fait la limite entre l'eau (ou le solvant) d'hydratation et l'eau (ou le solvant) libre. Les valeurs du potentiel zêta peuvent être calculées à partir de la mesure de mobilité électrophorétique, ce qui est d'un grand intérêt pratique. Il faut bien se rappeler que ce potentiel zêta ne mesure pas le potentiel total, mais seulement une partie de ce potentiel.

L'effet de l'existence de la double couche est de s'opposer à la rencontre des deux particules, et, par conséquent, de conférer une stabilité à la suspension colloïdale. L'épaisseur de la double couche et la densité de charge en surface des matières colloïdales dépendent de la concentration en ions de la solution et de la valence des ions. La stabilité de la suspension pourra donc être grandement affectée par l'addition d'ions à la suspension.

En résumé, les forces qui tendent à stabiliser une particule sont l'hydratation (qui forme une coquille protectrice de molécules d'eau) et la double couche électrique (qui provoque un potentiel électrique, d'où répulsion des particules).

\section{FACTEURS D'INSTABILITÉ}

Ce sont les forces qui ont tendance à déstabiliser la suspension, c'est-à-dire à la coaguler, selon le terme employé en traitement d'eau. L'un des premiers facteurs généralement invoqué est le mouvement brownien. En effet, les particules colloïdales - et principalement celles de dimension inférieure à $100 \mathrm{mu}$ - sont en continuel mouvement, se bombardant les unes les autres. L'énergie nécessaire à ce mouvement vient des collisions avec les molécules d'eau, et est en relation directe avec la température: l'énergie croît avec la température. Ce mouvement augmente la probabilité de collision entre les particules, mais il n'est cependant pas suffisant pour amener la déstabilisation complète de la suspension. D'autre part, pour les grosses particules, le mouvement brownien devient un facteur négligeable. On peut par contre augmenter les chances de collision entre particules en augmentant le mélange et en créant des zones de turbulence.

D'autres forces d'attraction entre particules sont les forces de Van der Waals, qui sont des forces de cohésion entre atomes. Bien que ces forces soient généralement considérées comme agissant à l'échelle des atomes, on considère que l'effet d'agrégation des particules colloïdales peut ressortir des mêmes forces.

Si les forces électriques de répulsion entre les particules sont suffisamment réduites pour permettre le contact de particules à particules, les forces de Van der Waals maintiendront les particules liées entre elles, conduisant à l'agglomération progressive des particules.

Comme la mesure du potentiel zêta est possible en étudiant la mobilité électrophorétique des particules, on est conduit à rechercher une corrélation entre le potentiel zêta et la déstabilisation ou coagulation. Les essais expérimentaux montrent qu’il n'est pas nécessaire de réduire lc potentiel zêta à 0 pour obtenir la déstabilisation, mais que la zone $P_{z}=-5 \mathrm{mV}$ conduit à des résultats appréciables. Cependant, il faut bien admettre que, plus le potentiel zêta est près de 0 , plus la déstabilisation semble complète.

En pratique, une relation empirique peut être établie, dans chaque cas, entre la qualité de la coagulation et le potentiel zêta, relation qui sera très utile dans le contrôle des conditions spéciffques de la coagulation.

Les forces dont on vient de parler sont uniquement des forces internes à un système colloïdal. Il est évident que des forces externes peuvent contribuer à modifier ces forces pour stabiliser ou déstabiliser les suspensions colloïdales.

Les études ont été commencées par Schultze (1882) et Hardy (1900), qui ont montré que la déstabilisation (= coagulation) est provoquée par une addition d'ions ayant une charge opposée à celle des colloïdes, et que l'action est d'autant plus intense que la charge électrique de l'ion est élevée: un ion bivalent est environ 30 à 60 fois plus efficace qu'un ion monovalent, et un ion trivalent est 700 à 1000 fois plus efficace qu'un ion monovalent. Les ions introduits neutralisent les charges de la particule, et le potentiel zêta de la particule s'annule.

On peut également obtenir la coagulation par l'introduction d'un colloïde de charge opposée. En réalité, la charge électrique de la particule ne s'annule pas complètement par suite de la présence de la double couche: seul le potentiel zêta s'annule, et il reste la couche d'hydratation. Mais cette couche peut être suffisamment mince pour que les forces de Van der Waals permettent aux particules de s'accrocher. 
La réduction de l'épaisseur de cette double couche dépend de l'ion en cause, et particulièrement de sa valence.

En réalité, dans le traitement de l'eau, la théorie est difficilement applicable de façon simple: les colloïdes sont très hétérogènes quant à leur nature, leur taille, leur composition chimique.

La déstabilisation s'effectue habituellement par addition de réactif's chimiques, dont l'ionisation fait apparaitre des charges électriques susceptibles d'annuler celles des collöides et d'éliminer les forces répulsives. Les sels minéraux à cations polyvalents (principalement fer et aluminium) ont été employés comme coagulants depuis les dernières années du siècle dernier et sont toujours largement utilisés dans les traitements de coagulation d'eau.

La dose d'électrolyte nécessaire peut se déterminer soit par électrophorèse, soit par des essais de floculation.

\section{1. Électrophorèse}

Cette technique consiste à déterminer la charge des particules colloïdales (potentiel zêta) en mesurant leur vitesse de déplacement lorsqu'elles sont soumises à l'action d'un champ électrique.

L'appareil utilisé pour cette détermination (Zêtamètre) comprend un boîtier de contrôle, une cellule électrophorétique, un dispositif d'éclairage, un microscope binoculaire qui permet l'examen de particules dont les dimensions sont voisines du micron. Un oculaire micrométrique et un compte-secondes permettent le contrôle de la vitesse de déplacement.

Le déplacement des particules, rendues visibles par effet Tyndall, s'observe dans le tube de liaison de la cellule.

Les déterminations sont effectuées d'abord sur l'eau brute, puis avec des doses croissantes d'électrolyte, en mesurant la vitesse de déplacement des particules collö̈dales dans chaque cas.

Des abaques donnent, en fonction de cette vitesse et de la température, le potentiel zêta des particules colloïdales. Ceci permet de tracer une courbe représentant l'évolution du potentiel (exprimé en millivolts) en fonction de la dose d'électrolyte. Le transport de l'eau, du lieu de prélèvement au laboratoire, n'affecte pas Ie résultat de l'étude électrophorétique.

\section{Essais de floculation}

Il est important de faire ces essais à une température voisine de celle que possèdera effectivement l'eau au cours de son traitement industriel.

En premier lieu, on expérimente un seul réactif à des doses différentes; si le résultat n'est pas suffisant, on recommence un nouveau test en répétant le traitement qui a donné le meilleur résultat dans le premier essai et en expérimentant un autre traitement en parallèle.

Il est nécessaire d'utiliser un floculateur permettant d'agiter simultanément, à une vitesse bien déterminée, le liquide contenu dans une série de béchers.

Pour que les résultats soient comparables, il faut en effet que la vitesse de rotation soit identique dans tous les béchers.

Cet essai est effectué pendant 20 minutes. On note les renseignements suivants:

a) dose de réactifs; b) aspect de la floculation;

c) $\mathrm{pH}$ après floculation.

Pour les meilleurs résultats obtenus, on complètera ces indications par les renseignements suivants:

- pourcentage de boues après décantation de $1 / 2$ heure;

- vitesse de sédimentation diffuse;

- coefficient de cohésion de la boue, ou vitesse de décantation en piston;

- oxydabilité au permanganate;

- couleur et turbidité de l'eau filtrée;

- Mesures particulières au traitement étudié.

\section{Examen de la sédimentation}

L'étude électrophorétique et l'essai de floculation ne suffisent pas pour transposer les résultats à une échelle industrielle, la question primordiale étant de savoir à quelle vitesse il sera possible de faire fonctionner un décanteur. Il y a donc lieu de procéder en outre à un examen de la sédimentation.

Deux cas peuvent se présenter:

- Au cours d'un essai de laboratoire, on peut obtenir une floculation relativement peu abondante, de telle sorte que, si on laisse au repos l'eau floculée, chaque particule se dépose comme si elle était seule, certaines à grande vitesse, d'autres à vitesse plus faible.

Le liquide se clarifie graduellement, un dépôt se forme au fond du bécher, c'est ce qu'on appelle une sédimentation libre ou diffuse.

- Dans l'autre cas, le liquide floculé est très trouble et la sédimentation se produit sur l'ensemble des particules floculées, laissant apparaître à la partie supérieure du bécher un liquide clair au-dessus d'une couche boueuse; c'est ce que l'on appelle la sédimentation freinée, ou en piston, qui, pratiquement, ne se produit que lorsqu'on traite des liquides très riches en matières floculables.

Les mesures à effectuer seront légèrement différentes dans l'un et l'autre cas.

\section{Schéma général de l'épuration}

Dans leur grande majorité, les eaux à épurer contiennent des colloïdes et les chaînes de traitement comporteront en général une floculation. Il est évidemment possible de concevoir l'utilisation de deux systèmes adjacents, par exemple floculateur et décanteur. Cependant, la mise au point d'appareils intégrés a permis l'obtention de performances remarquables: les décanteurs floculateurs modernes autorisent l'épuration à des vitesses de plusieurs $\mathrm{m}^{3} / \mathrm{m}^{2} . \mathrm{h}$, même avec des concentrations importantes. Les rencontres entre particules floculées, qui permettront leur grossissement, donc leur alourdissement, et une plus grande vitesse de sédimentation, seront d'autant plus fréquentes que la concentration dans l'eau sera plus élevée.

L'idée de base, pour la réalisation de décanteurs à hautes performances, est le renforcement de la concentration en conservant, dans le liquide, un fort pourcentage de boues formées antérieurement. La floculation des colloïdes se fait 
à la surface des flocons anciens et l'on obtient ainsi un précipité volumineux et dense.

Sans entrer dans le détail des réalisations technologiques, signalons simplement deux grandes familles de décanteurs floculateurs modernes:

- Les appareils à circulation de boues, où il y a séparation de l'eau traitée et des boues par décantation, et retour de celles-ci dans une zone de mélange comportant une agitation mécanique ou hydraulique et une injection de réactifs chimiques assurant la bonne coagulation et la valeur adéquate du $\mathrm{pH}$.
- Les appareils à lit de boues. La boue y est maintenue en expansion à la partie basse du décanteur et l'eau la traverse de bas en haut d'une façon aussi régulière et uniforme que possible. Le brassage, qui favorise la floculation, résulte des mouvements tourbillonnaires suffisamment doux créés par l'injection d'eau brute à laquelle ont été préalablement mélangés les réactifs nécessaires.

L'intérêt de tels appareils est tel que la filtration est plutôt devenue un traitement de finition placé, si nécessaire, après la décantation.

\section{Discussion}

Président: M. le Professeur A. Fortier

M. le Président remercie $M$. Treille pour son très intéressant exposé qui montre notamment les artifices utilisés pour éliminer même les collö̈des, par précipitation gravitaire. It ouvre ensuite la discussion.

Sur une question de $M$. Bonnin, $M$. Treille précise que les poly-électrolytes «ne sont pas encore allorisés» en France pou le traitement des eaux potables; dès maintenant, certains sont autorisés en Angleterre et aux Etats-Unis pour ce genre de traitement. En France, les Services de l'Hygiène préfèrent attendre davoir de connaissances plus précises sur certains polymères à longue chain qui sont soupçonnes davoir des actions cancérigènes ou tératogènes.

M. Françols désirerait obtenir des précisions sur le mécanism d'élimination des impuretés à la surface d'un «flotateur».

C'est l'un des problèmes rencontrés dans ies procédés de flottation, répond $M$. Treille, que de maintenir la boue en surface pour qu'un système de raclage permette de la recueillir grâce à un système di pales mobiles. Pour que la boue reste en surface suffisamment longtemps, il est possible qu'il soit nécessaire d'injecter à la partie inférieure du bassin un poly-électrolyte ou un corps permettant de rendre moins hydrophile le floc et l'empêchant de retomber. Cela dépend beaucoup du type de la pollution, mais ce n'est pas fondamental.

Bien que cela sorte quelque peu du thème de cette session, le conférencier attire l'attention sur l'importance et le coût des systèmes de traitement des boues des stations d'épuration. Dans certains cas, les installations de traitement de ces boues sont plus importantes et plis conteuses que les installations de traitement d'eau proprement dites.

Le «flottateur» présente l'intérêt de sortir en quelque sorte la boue de l'eau. Dans le décanteur, au contraire, la boue est au fond de l'eau et toujours en contact étroit avec celle-ci. Dans le décanteur, on peut extraire de la boue contenant $3 \mathrm{~g}$ de matière sèche par litre; dans le flottateur, on peut obtenir des boues directement pelletables c'est-à-dire contenant $100 \mathrm{~g}$ de matière sèche par litre.

Il faut penser au traitement ultérieur qui doit permettre de dispose: de la boue pour l'évacuer vers une décharge ou l'envoyer à l'incinération. Pilus cette boue est sèche, moins le problème est difficile. Il peut donc être avantageux de compliquer quelque peu linstallation d'épuration en vue de simplifier le système de traitement des boues.

Pour la séparation des colloïdes, demande M. Françols, ne pellt-on envisager un traitement purement électrostatique un peu analogue à ccux utilisés pour le dépoussiérage des gaz?

Nous avons effectivement à l'étude, répond $M$. Treille, un traitement électrique; mais il semble, en définitive, que l'on n'arrive à une bonne «coagulation» qu"en utilisant des électrodes «consonmables $\$$ en fer ou en aluminium, lesquelles fabriquent des hydroxydes complexes de fer ou d'aluminium qui constituent des porteurs de charge électrique dont il convient d'ajuster l'action pour assurer la bonne efficacité du procédé.

Est-ce que vous n'êtes pas quelquefois gêné par les mouvements de convection naturelle qui peuvent se produire dans les bassins"? interroge $M$. le Président.

L'action du soleil en particulier, jépond M. TREILlE, peut provoquer des différences de tempéraure entre les régions exposées et celles à l'ombre; dans les décanteurs de très grande dimension cela peut provoquer des mouvements de convection et des écoulements parasites dont la vitesse est du même ordre que la vitesse de chute de cerlaines particules.

L'expérience montre que l'addition de poly-électrolytes ou do chatix peut a:tgmenter cette vitesse de chute et neutraliser l'effet des courants de convection. Dans certains cas difficiles, on peut être amené à injecter un gramme, et même deux grammes, de sulfate d'alumine par litre d'ea: à traiter et cette quantité de sel va se retrouver dans les boues. Aussi, importe-t-il d'étudier l'ensemble du problème «épuration de l'eau + traitement des boues» afin d'optimiser les frais d'investissement et d'exploitation de l'ensemble.

A titre d'exemple, M. Trenle cite le traitement, all port de Marseille, des boues provenant des hydrocarbures séparés de l'eau dans les stations de «déballastage» des pétroliers ou analogues. L'incinération de telles boues - qui demande un appoint de fuel est devenue si coûteuse qu'il est possible que, dans ce cas, le choix de la méthode d'épuration des eaux soit presque imposé par le coût d'élimination des résidus du traitement.

M. Misson s'interroge sur la possibilité d'utiliser de tels résidus comme engrais pour l'agriculture.

Il en résulte un échange de vues dans lequel interviennent, outre M. Treille, M. Minart (SOGREAH), M. Martin (Agence de Bassin Adour-Garonne), M. Bouteaud (Renardet Engineering) M. Prompsy (Sté Lyonnaise des Eaux et de l'Eclairage) et M. Serpaui (Alsthom-Techniques des Fluides).

Il en ressort que, sauf cas particulier, cette utilisation n'est guère rentable: cô̂t du transport, présence dans les boues de réactï̌s d'épuration (sulfate d'alumine, sels ferriques), de métaux lourd: (plomb, mercurc, etc.), de germes pathogènes, de pesticides, de détergents, etc. Le recyclage de coagulants (sels de fer ou daluminium), la récupération ou l'élimination de certains corps (composés azotés ou phosphorés) peut conduire dans lavenir à reconsidére ce problème.

En raizon des exigences de l'horaire, $M$. le Président clôt la discussion en remerciant tous ceux qui l'ont animée et donne la parole à M. Minari pour l'exposé de sa communication. 
Abstract

\section{Gravity separation of water and solids} in suspension

Water recycling in industry is not basically different from water purification for human consumption or industrial purposes.

The first phase, if not the only phase of the purification process, consists in the removal of suspended solids. Average particle size varies considerably even for a given type of pollution (see Table 1) and natural elimination by gravity takes too long below a certain particle size.

Pollution by suspended solids may be characterized as follows:-

a) by content, i.e. suspended solids concentration;

b) by turbidity.

Units have been determined for each case, as well as measurement methods whereby process efficiency can be established.

Solid particles are extracted by two different methods:-

-By gravity: flotation or sedimentation;

- By filtration or straining (distinction between macro, micro and ultra-filtration).
These methods are unsuitable for direct elimination of colloids, for the following reasons:-

-Retention times are inconsistent with equipment operating conditions;

-Prohibitive cost.

The elimination or colloids requires preliminary action to eliminate stability factors and allow collection of much coarse colloid particles for subsequent separation from the water by the above methods.

Tests have been devised for establishing how to best deal with colloids. Two main types of test are electrophorelic measurement and flocculation tests.

As pollution is often at least partly colloidal, most purification plants must include a fiocculation-coagulation stage followed by one or more separation systems.

As regards sedimentation, technological developments have resulted in the design of such widely-used compact units as sludge recircuiation and sludge-bed equipment whose efficiency is such that filtration has often become the final process completing clari-flocculator action. 